\title{
INTERMOLECULAR VIBRATIONAL MODES OF CRYSTALLINE POLY-(R)- 3-HYDROXYBUTYRATE OBSERVED AT TERAHERTZ FREQUENCIES INVESTIGATED BY MOLECULAR DYNAMICS
}

\author{
Md. Khorshed Alam ${ }^{1 *}$, Md. Saif Ishtiaque ${ }^{1}$, Md. Imran Hossain ${ }^{1}$, Hiromitsu Takaba ${ }^{2}$ \\ ${ }^{1}$ Department of Physics, University of Barishal, Kornokathi, Barishal-8200, Bangladesh \\ ${ }^{2}$ Department of Environmental Chemistry and Chemical Engineering, School of Advanced \\ Engineering, Kogakuin University, 2665-1 Nakano, Hachioji, Tokyo 192-0015, Japan.
}

*Corresponding authore-mail: khorshed_du@yahoo.com

Received on 08.09.2019, Revised received on 02.12.2019, Accepted for publication on 11.12.2019

\begin{abstract}
Terahertz (THz) absorption spectra of poly 3-hydroxyalkanoates (PHB) for different conformations were investigated using molecular dynamics (MD) method. Temperature-dependent THz absorption spectra of PHB were measured over a temperature range of $10 \mathrm{~K}$ to $200 \mathrm{~K}$. Peaks around $(2.4-2.6 \mathrm{THz})$ and $(3.1-3.2 \mathrm{THz})$ were observed due to vibrational transition of $\mathrm{PHB}, 1$ stpeaks are polarized perpendicular to $\mathrm{c}(\perp)$ axis (along a, b axis) and next peaks are oriented parallel to the $\mathrm{c}(/ /)$ axis. The peak around $(2.4-2.6 \mathrm{THz})$ was assigned due to vibrational transition of $\mathrm{PHB}$ and $\mathrm{C}-\mathrm{H}{ }^{\cdots} \mathrm{O}=\mathrm{C}$ hydrogen bonds are oriented perpendicular to the $\mathrm{c}(\perp)$ axis. We have also investigated orientation of the intermolecular hydrogen bonds by MD simulation and confirmed that it was mainly along the $\mathrm{b}$ axis of PHB. THz absorption spectra shifted to the lower frequencies and noticed widening of the absorption peaks that visualized from characteristics of peaks within creasing temperature, which is well reproduce of experimental observation.
\end{abstract}

Keywords: THz, PHB, MD simulations, Hydrogen bonding.

\section{INTRODUCTION}

Recently, THz frequency has received an increasing interest from chemistry, material sciences and physics. Potential applications in the above fields [1-5] recent new developments in system engineering led to a wider implementation of this non-destructive, non-contact technique in laboratories, industrial production lines, and security portals. $\mathrm{THz}$ frequency radiation possesses a unique combination of desirable properties for non-destructive materials imaging and spectroscopy that has caught researchers' attention [4,6]. Many materials exhibit characteristic spectral features in the $\mathrm{THz}$ frequency range (particularly $>1.0 \mathrm{THz}$ ), enabling $\mathrm{THz}$ spectroscopy to be used as a tool to uniquely identify chemical species. In addition to addressing intra-molecular vibrational modes, THz spectroscopy can also excite intermolecular vibrations [7-11]. This gives $\mathrm{THz}$ spectroscopy the potential to provide both chemical and structural information. Indeed, THz-TDS has been shown to be a particularly sensitive technique for studying the structural dynamics of crystal forms, providing additional information for sample analysis. Furthermore, it is worth noting that THz-TDS can provide complementary spectral information to Raman spectroscopy, owing to the different selection rules governing IR and Raman transitions. THz spectra of some polymers have been measured by THz-TDS and the observed spectra yielded information about higher order and dynamics [12-14]. Since THz-TDS detects the THz electric field coherently, the signal is free from thermal background noise, and the obtained spectra show a significantly large 
dynamic range in the frequency region below 3.0 THz [15]. Many applications have become possible with the help of characteristic spectra (so-called fingerprint spectra) in the $\mathrm{THz}$ frequency range, such as the illicit drugs detection [16,17] different explosives detection [18] and imaging of cultural heritage artifacts [10]. Most of those purposes use the fingerprint spectra of organic molecules to identify materials $[9,11,19]$.

As we know that the origin of such spectra is skeletal motions coupled with intermolecular vibrations [20], information regarding inter molecular interactions, such as hydrogen bonding, can be effectively probed by $\mathrm{THz}$ spectroscopy. Hydrogen bonding is one of the dominant interactions that determine the higher order conformations of large molecules, such as proteins, DNA molecules, and polymers. Therefore, THz spectroscopy is expected to be useful in understanding how large molecules form a higher order conformation [21-25]. Far-infrared (FIR) absorption spectroscopy and low-frequency Raman scattering results obtained for polymers have been studied since the 1970s [26]. Sometimes, polymers show broad absorption features in the FIR region due to skeletal motions of polymer chains and vibrational motions of side groups [27, 28]. Some polymers exhibit low-frequency vibrational bands for intermolecular interaction in the FIR and low-frequency Raman spectra [29-31]. Wietzke et al. recently studied the temperature-dependent spectra of high-density polyethylene [32] and polyvinylidene [33] by THz-TDS [21,22]. They observed that the thermal gradient of crystal lattice modes changes at the glass transition temperature, which indicates a strong interaction between the amorphous and crystalline domains in the polymer [21]. At lower frequencies, vibrational peaks are observed due to hydrogen bonding and such peaks are observed in the $\mathrm{THz}$ spectra of polymers with hydrogen bonds, such as nylon [12], poly (methacrylic acid) [30], poly (lactic acid) [34] and poly PHB [23,24]. By using THz spectroscopy, we might obtain detailed information about how hydrogen-bonding results in the higher order conformation of polymers. PHB is produced by natural bacteria and has been attracting keen interests because of its good thermal and mechanical properties and water resistance [35,36]. Its physical properties have been a subject of active research. There are some recent studies on PHB and its co-polymers by infrared (IR) spectroscopy [37,38], X-ray diffraction [38,39], Raman spectroscopy[39] and differential scanning calorimetry [39] and they demonstrated the contribution of $\mathrm{C}=\mathrm{O} \cdots \mathrm{H}-\mathrm{C}$ hydrogen bonds between two helices to the crystal morphology.

Yamamoto et al. recently performed quantum mechanical interpretation of intermolecular vibrational modes of PHB for low frequency Raman and $\mathrm{THz}$ spectra [40]. They successfully assign that the intermolecular vibrational bands due to $\mathrm{CH}_{3}$ and $\mathrm{O}=\mathrm{C}$ groups of PHB appearing in both of the experimental low frequency Raman and THz (FIR) absorption spectra and using QM interpretation provide realistic spectra of the PHB with the explicit corrections for the intermolecular interactions among polymer chains. $\mathrm{THz}$ spectroscopy may also provide new insight into the relationship between hydrogen bonding and higher order conformation, which would be directly reflective of the physical properties of PHB. The vibrational modes is subject to uncertainties if it is based only on the experimental results and the spectral simulation based on theory require to be performed to confirm such phenomena. However, accurate calculation using DFT based simulations requires long calculation time and some limitations for large molecules such as polymers.

In the present study, we have applied MD method to simulate THz spectra of PHB. The MD method was used to calculate the vibrational spectrum of large molecular systems consist of several thousand atoms within reasonable computational cost. The MD simulation is faster than density functional theory (DFT) based MD simulations and quantum mechanical methods. By 
using the MD simulation tools, we could observe the conformational change in the polymer structure and consider the temperature effect during the simulation. Here temperature dependency and force field effect on the $\mathrm{THz}$ absorption spectra were investigated. We have also visualized the atomic vibration of polymers, direction of hydrogen bonding within lamellar crystal of PHB and investigated the cell size effect on the THz absorption spectra.

\section{COMPUTATIONAL METHODOLOGY}

The chemical structure of PHB is shown Fig. 1 (a). Our MD simulation system contains $4 \times 2 \times 4$ time of orthorhombic unit cell of PHB as shown in Fig.1 (b-d). MD simulation performed with canonical ensemble (NVT) or isothermal-isobaric ensemble (NPT) [41]. In all MD simulations, temperature and pressure were controlled by Andersen thermostat and Berendsen's method [42]. MD calculations were performed for $100 \mathrm{ps}$ at temperature $300 \mathrm{~K}$. Suitable force field plays an important role in achieving desired accuracy of simulation results. To select accurate force field, we employed several force fields. Consistent Valence Force Field (CVFF) [43], Polymer Consistent Force Field (PCFF) [44,45], Condense phase Optimized Molecular Potentials for Atomistic Simulation Studies (COMPASS) [46] and UNIVERSAL force field [47] for $\mathrm{THz}$ absorption spectra of PHB and we adopted CVFF as the basis of our potential function for detail analysis.

(a)<smiles>C[C@@H]1CC(=O)CC(C)(C)C(CP)O1</smiles>

(c) (b)

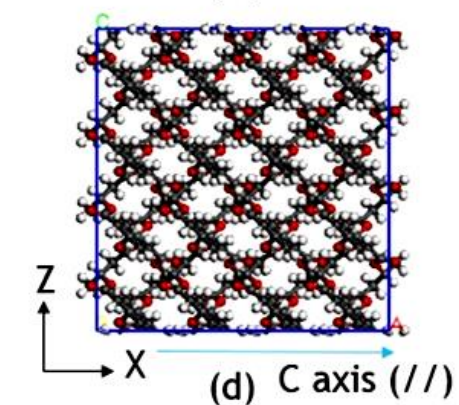

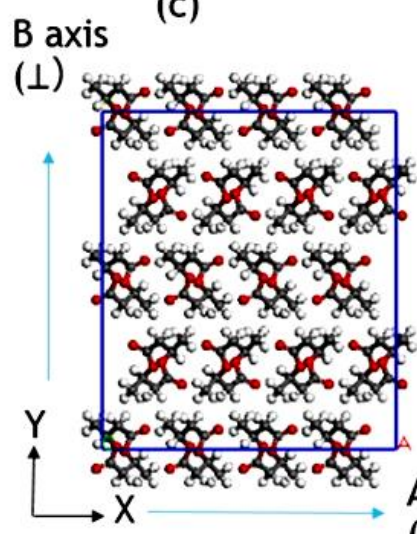

( 1 )

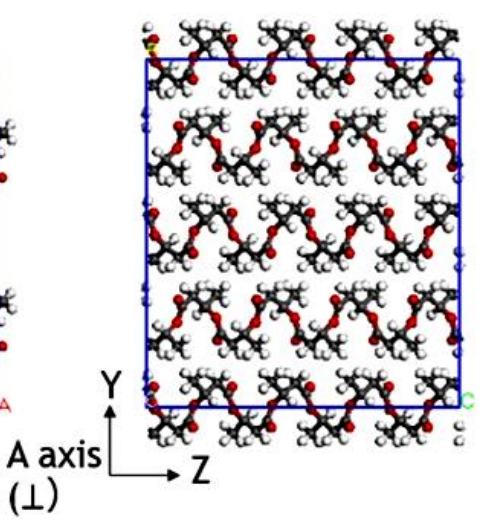

Fig.1. (a) Chemical structure of PHB (b) Optimized structure of PHB, polarization direction along c axis, (c) Intermolecular interactions among PHB along the b axis, (d) Intermolecular interactions among PHB along the a axis. 
The molecular dynamics simulation method is based on Newton`s second law or the equation(1):

$$
\sum_{i \neq i}^{N} \boldsymbol{F}_{i}=m \boldsymbol{a}_{i}=m \frac{d^{2} \boldsymbol{r}_{i}}{d t^{2}}
$$

Where $F_{i}$ is the force exerted on particle $i, \mathrm{~m}$ is the mass of particle and $a_{i}$ is the accelerated of particle $i$. The force can also be expressed as the gradient of the potential energy.In this simulation, to obtain the spectrum of the polymer structure model, we follow the Fourier transform of the autocorrelation function of the dipole moment given as equation (2):

$$
C(t)=\left\langle\mu\left(t_{0}\right) \cdot \mu\left(t_{0}+t\right)\right\rangle
$$

Here we represent $\mu_{i}(t)$ and the dipole moment of atom $i$ at time $t$ obtained by the molecular dynamics simulation method and obtained the following (equation 3 ) autocorrelation function:

$$
C(t)=\left\langle\sum_{i} \mu_{i}\left(t_{0}\right) \cdot \sum_{j} \mu_{j}\left(t_{0}+t\right)\right\rangle
$$

The autocorrelation function obtained in the following form (equation 4) used to analyze vibration distribution:

$$
\mathrm{I}(\omega)=\frac{1}{2 \pi} \int e^{-i t \omega}\langle\mu(0) \cdot \mu(t)\rangle \mathrm{dt}
$$

CVFF is fitted to reproduce small organic crystals and gas phase structures. It handles peptides, proteins and a wide range of organic systems. It is primarily intended for studies of structures and binding energies, although it predicts vibrational frequencies and conformational energies reasonably well. The functional of CVFF form can be illustrated as followsin (equation 5):

$$
\begin{aligned}
E_{p o t} & =\sum_{b} D_{b}\left[1-e^{-a\left(b-b_{0}\right)}\right]^{2}+\sum_{\theta} H_{\theta}\left(\theta-\theta_{0}\right)^{2}+\sum_{\phi} H_{\boldsymbol{\phi}}[1-\operatorname{scos}(n \boldsymbol{\phi})] \\
& +\sum_{x} H_{x} x^{2}+\sum_{b} \sum_{b^{\prime}} F_{b b^{\prime}}\left(b-b_{0}\right)\left(b^{\prime}-b_{0}^{\prime}\right)+\sum_{\theta} \sum_{\theta^{\prime}} F_{\theta \theta^{\prime}}\left(\theta-\theta_{0}\right)\left(\theta^{\prime}-\theta_{0}^{\prime}\right) \\
& +\sum_{b} \sum_{\theta^{\prime}} F_{b \theta^{\prime}}\left(b-b_{0}\right)\left(\theta-\theta_{0}\right)+\sum_{\phi} F_{\phi \theta \theta^{\prime}} \cos \left(\theta-\theta_{0}\right)\left(\theta^{\prime}-\theta_{0}^{\prime}\right)+\sum_{x} \sum_{x^{\prime}} F_{x x^{\prime}} x x^{\prime} \\
& +\sum_{\varepsilon} \varepsilon\left[\left(\frac{r^{*}}{r}\right)^{12}-2\left(\frac{r^{*}}{r}\right)^{6}\right]+\sum \frac{q_{i} q_{j}}{\varepsilon r_{i j}} \\
& 11
\end{aligned}
$$

The energy terms were divided into three categories: the bonded energy terms, the cross terms and the non-bonded energy terms. Where the first two terms for the binding force, $3^{\text {rd }}$ term is dihedral angle, $4^{\text {th }}$ term is out of plane energy term described for as a harmonic function, terms 5 to 9 are cross terms interactions included the dynamic variations among the bond stretching, bending and 
torsion angle rotation and terms 10 and 11 represent non-bonded terms with the Coulombic interaction between atomic charges and the van der Waals interactions among the PHB.

\section{RESULTS AND DISCUSSION}

To investigate the intra and intermolecular vibrational modes among the PHB, we conducted a MD simulation using Forcite module[48, 49].In Fig.1(a)shows the chemical structure of PHB, which has been determined by the wide-angle $\mathrm{X}$-ray diffraction measurement [50]. It was reported that the unit cells of PHB belong to the orthorhombic system, $P 2_{1} 2_{1} 2_{1}\left(D_{2}{ }^{4}\right),\left(\alpha=\beta=\gamma=90^{\circ}\right)$ with $a=5.76 \AA, b=13.20 \AA, c=5.96 \AA$ (fiber repeat) [50, 51]. In our system we have considered super cell $(\mathrm{a} \times 4, \mathrm{~b} \times 2$, and $\mathrm{c} \times 4$ ) keeping the same lattice parameter. In Fig.1 (b, c and d) shows optimized crystalline structures of PHB.

(a)

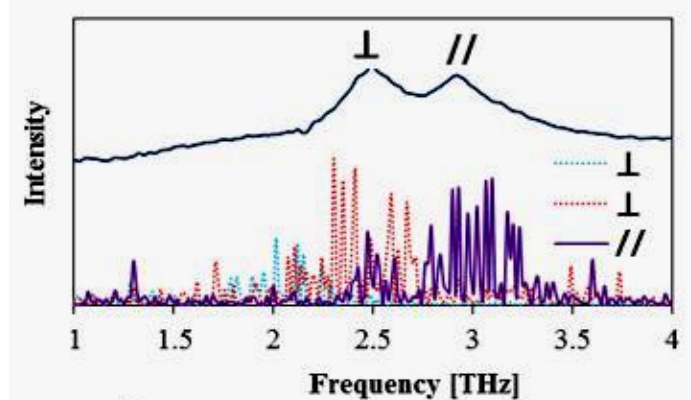

(c)

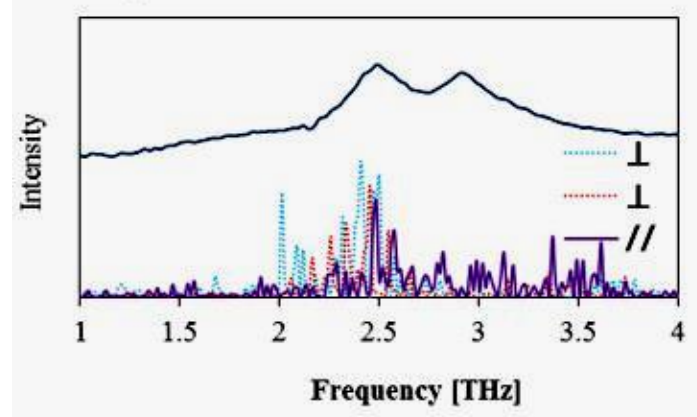

(b)

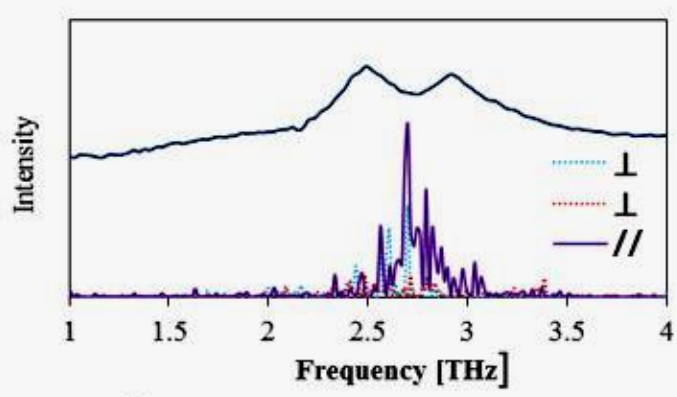

(d)

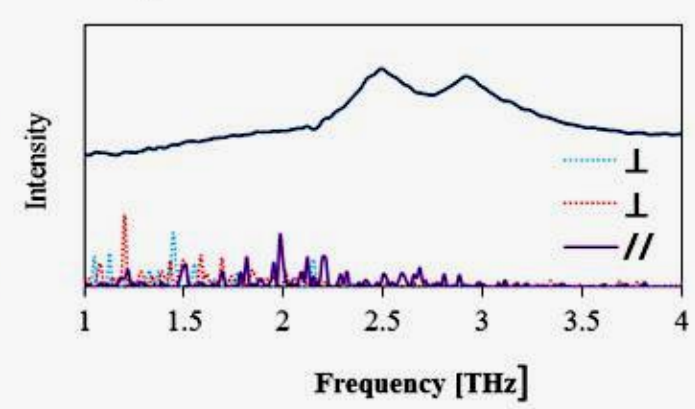

Fig. 2. THz absorption spectra of PHB of in the frequency region of 1.0-4.0 THz for different force field at temperature $300 \mathrm{~K}$. (a) absorption spectra for CVFF, (b) absorption spectra for PCFF, (c)absorption spectra for COMPASS and (d)absorption spectra for UNIVERSAL.

One of the major challenges in polymer folding simulations is choosing an appropriate force field [52]. Selection of suitable force field plays an important role achieving desired accuracy of simulation results. Force field modifications have led to improved agreement between MD simulations and experimental data. To choose accurate force field for MD simulation we have 
applied four types of force fields to calculate the THz absorption spectra of PHB. In Fig. 2 shows the calculated THz absorption spectra of crystalline PHB. In Fig.2 (a), (b), (c) and (d) are the absorption spectra of crystalline PHB using CVFF, PCFF, COMPASS and UNIVERSAL force fields respectively, at temperature $300 \mathrm{~K}$. In the Fig.2 (a) THz spectra for the CVFF force field at $300 \mathrm{~K}$ there are two peaks observed around $2.4 \mathrm{THz}$ and $3.1 \mathrm{THz}$, which is the well, reproduce of experimental THz absorption spectra [14]. As in our system CVFF shows the best performance among the four types of force field, so we have discussed $\mathrm{THz}$ adsorption spectra, vibrational dipole moment and vibrational motion of PHB crystal are discussed using CVFF force field.

Table 1: Calculated atomic charges of PHB using different force field.

\begin{tabular}{llll}
\hline Atom & \multicolumn{3}{c}{ Charge [e] } \\
\cline { 2 - 4 } & CVFF & PCFF & COMPASS \\
\hline c1 (-CH-) & 0.05 & 0.172 & 0.107 \\
c2 $\left(-\mathrm{CH}_{2}-\right)$ & -0.20 & -0.106 & -0.106 \\
c3 $\left(-\mathrm{CH}_{3}\right)$ & -0.3 & -0.159 & -0.159 \\
o & -0.18 & -0.396 & -0.272 \\
ó & -0.38 & -0.531 & -0.450 \\
h & 0.10 & 0.053 & 0.053 \\
\hline
\end{tabular}

We have examined the atomic charge of PHB for different force fields as shown in Table 1. The calculated charge of hydrogen atom is 0.10 e for CVFF force field. On the other hand, the charge value of hydrogen is $0.053 \mathrm{e}$ for both PCFF and COMPASS force fields. We have noticed that CVFF charge of hydrogen is almost 2 times larger than those of PCFF and COMPASS charges. Calculated charge value of ó in $\mathrm{O}=\mathrm{C}$ (which take part in hydrogen bonding among $\mathrm{PHB}$ ) is -0.380 e for CVFF, -0.531 e for PCFF and -0.450 e for COMPASS. Although CVFF charge of oxygen is less negative than those of other two force fields but if we consider charge value of this ó-h pair, the electrostatic force will be larger for CVFF than other two that indicates stronger intermolecular interactions among crystalline PHB. In Fig.1 (b-d) shows the optimized crystalline PHB structures of different orientation to show the intra-and intermolecular interactions among the PHB chains. In Fig.1 (b) main polarization direction of PHB parallel to the $c(/ /)$ axis. In Fig.1 (c) and (d) main polarization direction are perpendicular to the $\mathrm{c}(\perp)$ axis which means along a and $\mathrm{b}$ axis respectively. 


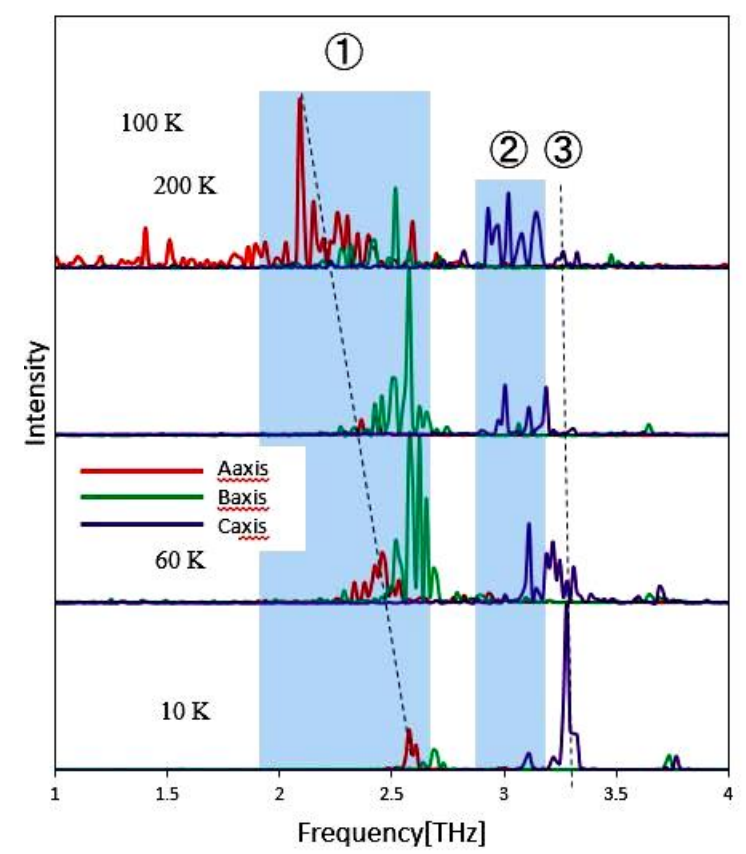

Fig. 3. THz absorption spectra of PHB using MD simulation over temperature range (10-200 K).

Fig. 3 shows the temperature dependent THz absorption spectra of PHB that obtained from our MD simulation. Since low-frequency vibrational motions originate from intra-molecular motions strongly coupled with intermolecular motions. In Fig. 3 shows the absorption spectra that originate from the vibrational motion of different polarization direction. Peaks of the different polarization (A, B and $\mathrm{C}$ axis) direction denoted as red, green and violet colour respectively. At $10 \mathrm{~K}$ there is some adsorption peaks were observed between $2.5 \mathrm{THz}$ to the $3.8 \mathrm{THz}$. Major peaks at temperature $10 \mathrm{~K}$ were visible around $(2.5-2.7 \mathrm{THz})$ and $(3.1-3.4 \mathrm{THz})$. At temperature $60 \mathrm{~K}$ the peaks position $(2.5-2.7 \mathrm{THz})$ shifted to lower frequencies range $(2.3-2.5 \mathrm{THz})$. On the other hand next peaks position (3.1-3.4 THz) shifted to peaks around $3.2 \mathrm{THz}$. At the $100 \mathrm{~K}$ we observed same behaviour with higher temperature that peaks around $2.3 \mathrm{THz}$ shifted to lower frequencies while the peaks between (3.1-3.4 THz) shifted to (2.95-3.1 THz). Finally, the highest temperature (200 $\mathrm{K})$ that we considered in our simulation, the vibrational peaks between $(2.5-2.7 \mathrm{THz}$ at $10 \mathrm{~K})$ spectral seems to overlap each other and shifted to lower frequencies around (2.1-2.4 THz). The vibrational peaks (3.1-3.4 THz at $10 \mathrm{~K}$ ), shifted to lower frequencies $2.9 \mathrm{THz}$ but the peaks around $3.8 \mathrm{THz}$ almost disappear at higher temperature. Thus, temperature dependency of $\mathrm{THz}$ spectra by our MD simulation shows the almost same tendencies as we observed by the experimental studies [14]. In experimental report shows that peak position shifted linearly to lower frequencies at higher temperature and the line widths also increases with temperature. In our simulation results also shows that at high temperature the peaks along the $b$ axis overlapped with a axis and the number of adsorption peaks increasing with the temperature which ultimately generates widen peak at higher temperature as shown in Fig.3 which has similarity with experimental report [14]. 
Hoshina et al. from their terahertz time-domain spectroscopy reported that peak around $2.5 \mathrm{THz}$ is due to the vibrational dipole transition moment perpendicular to the c-axis and the peak around $2.92 \mathrm{THz}$ is due to the vibrational dipole transition moment parallel to the c-axis [12].

In our study, we also noticed the absorption peaks around $(2.5-2.7 \mathrm{THz})$ and the peaks around (3.1$3.4 \mathrm{THz}$ ) from the simulation of PHB. Absorption spectra originate around $(2.5-2.7 \mathrm{THz})$, due to vibrational interactions among the lamellar crystal of PHB through $\mathrm{C}-\mathrm{H}^{\cdots \cdots} \mathrm{O}=\mathrm{C}$ hydrogen bonds which are oriented perpendicular to the $\mathrm{c}(\perp)$ axis (parallel to the a, b axis) as shown in Fig.1(c) and (d) respectively. In the peaks around (3.1-3.4 THz) the vibrational modes of these peaks source are the intra-molecular interaction like spring motion along the c (//) axis as shown Fig.1 (b). In the higher temperature spectral feature is changing with the temperature changes. Peaks intensities along the different axis are not uniformly behave with the temperature because of the atomic vibration due to MD simulation as shown in Fig.3.

At temperature $10 \mathrm{~K}$ the peaks around $(2.5-2.7 \mathrm{THz})$ mainly originate from the intermolecular interactions, which polarized along a,b axis. On the other hand, peaks around $2.5 \mathrm{THz}$ at $60 \mathrm{~K}$ and $100 \mathrm{~K}$ peaks seems to be major contribution from the spectral lines along b axis. At temperature 10 $\mathrm{K}$ the peaks around (3.1-3.4 THz) arise due to the intermolecular interactions, which polarized completely along $\mathrm{c}$ axis and the peak intensities changing with temperature (Fig.3). At $10 \mathrm{~K}, \mathrm{THz}$ spectra noticed around $(2.5-2.7 \mathrm{THz})$ and $(3.1-3.4 \mathrm{THz})$ when temperature increase the $1 \mathrm{st}$ absorption peak shifts to $(2.4-2.6 \mathrm{THz})$ at temperature $60 \mathrm{~K}$ and $2.1 \mathrm{THzat}$ temperature at $200 \mathrm{~K}$. On the other hand, peaks around (3.1-3.4 THz) shifted to $(3.0-3.2 \mathrm{THz})$ at $100 \mathrm{~K}$ and $(2.9-3.1$ $\mathrm{THz}$ ) at temperature $200 \mathrm{~K}$ (Fig.3). This linearly shifting of absorption peaks position with temperature which also comparable to the experimental study [14]. From the Fig.3 we also noticed that the number of absorption peaks appeared in wide range of frequency with increasing temperature. At $10 \mathrm{~K}, 60 \mathrm{~K}$ and $100 \mathrm{~K}$ there was no any visible absorption spectra before $2.2 \mathrm{THz}$, 1st absorption peaks located around (2.5-2.7 THz) range. On the other hand, at $200 \mathrm{~K}$ we observed there are number of absorption peaks before $2.2 \mathrm{THz}$ and the 1stabsorption peaks located a wide range (1.0-2.5 THz) region. It is clearly indicate that the line width of the spectrum increasing with temperature which comply with experimental observation [14].

In Fig. 4 shows the $\mathrm{THz}$ absorption spectra at $300 \mathrm{~K}$ for different cell size along the $\mathrm{b}$ axis. Here we increase the cell size slightly along $b$ axis with keeping cell size unchanged of others direction. In Fig. 4(a) cell size of the system along the b axis was $26.30 \AA$ and the center of absorption peaks was located around $2.5 \mathrm{THz}$, (b) cell size along the $\mathrm{b}$ axis was $26.38 \AA$ and the center of absorption peaks was $2.45 \mathrm{THz}$ and (c) cell size along the b axis was $26.42 \AA$ and the center of absorption peaks was around $2.35 \mathrm{THz}$. We noticed here that peaks position was linearly shifted to the lower frequencies with increasing the cell size along the $\mathrm{b}$ axis. In previous section, we have already discussed that absorption peaks arise around (2.5-2.7 THz) originate due to the intermolecular $\mathrm{C}$ $\mathrm{H}^{\cdots \cdots} \mathrm{O}=\mathrm{C}$ hydrogen bonding along the $\mathrm{b}$ axis (perpendicular respect to the $\mathrm{c}(\perp)$ axis). Thus we conclude, from the $\mathrm{THz}$ absorption spectra that the cell size expansion along the $\mathrm{b}$ axis increases the distance between $\mathrm{C}-\mathrm{H}^{\cdots \cdots} \mathrm{O}=\mathrm{C}$ bonds and weakening of the $\mathrm{C}-\mathrm{H}^{\cdots \cdots} \mathrm{O}=\mathrm{C}$ interactions which is one of the important factor of shifting peaks to lower frequency. 


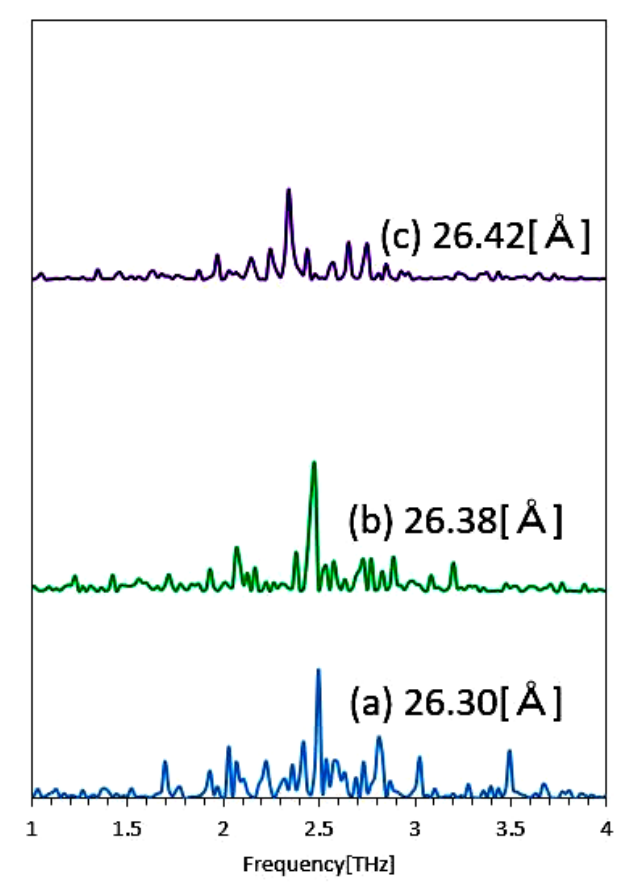

Fig. 4. THz absorption spectra of PHB for different cell size along $b$ axis.

\section{CONCLUSION}

In this paper, $\mathrm{THz}$ absorption spectra of PHB measured by the MD simulation using different force field. Visible differences were observed of $\mathrm{THz}$ spectra for crystalline $\mathrm{PHB}$ for different force field and the obtained $\mathrm{THz}$ spectra for CVFF is well reproduce of experimental observation. Temperature dependent $\mathrm{THz}$ absorption spectra of our study, peaks that originate from the intra and intermolecular interactions are polarized perpendicular to the $\mathrm{c}$ axis (along the $\mathrm{a}, \mathrm{b}$ axis) and shifts to the lower frequencies within creasing temperature. From the MD simulation, we could confirm $\mathrm{THz}$ absorption around $2.5 \mathrm{THz}$, originate due to the intermolecular interaction along a,b axis of PHB. Our MD calculations demonstrated details analysis of intermolecular interactions and polarization directions of atoms clearly in the large scale crystalline PHB, which can open new insight into hydrogen bonds in various polymers and other molecules including proteins.

\section{ACKNOWLEDGMENT}

This work was supported in part by the Kogakuin University, Japan providing computational facilities. 


\section{REFERENCES}

[1] P. U. Jepsen, D. G. Cooke and M. Koch, Laser Photon. Rev., 5, p-124, (2010).

[2] M. F. Kimmitt, J. Biol. Phys., 29, p-77, (2003).

[3] D. Mittleman, Springer-Verlag, Berlin, (2003).

[4] P. H. Siegel, IEEE Trans. Microwave Theory Tech., 50, p-910, (2002).

[5] J. P. Laib and D. M. Mittleman, J. Infrared, 31(9), p-1015, (2010).

[6] J. F.Federici, B.Schulkin, F. Huang, D. Gary, R. Barat, F. Oliveira and D.Zimdars, Semicond. Sci. Technol., 20, p-S266, (2005).

[7] M. Walther, B. M. Fischer and P. U. Jepsen, Chem. Phys., 288, p-261, (2003).

[8] P. U. Jepsenand S. J.Clark,Chem. Phys. Lett., 442, p-275, (2007).

[9] D.G. Allis, A.M. Fedor, T.M. Korter, J.E. Bjarnason and E.R. Brown,Chem. Phys. Lett., 440, p-203, (2007).

[10] A. G. Davies, A.D. Burnett, W. Fan, E.H. Linfield and J.E. Cunningham, Materialstoday, 11,8-26, (2008).

[11] N. Fuse, R. Sato, M. Mizuno, K.Fukunaga, K. ItohandY. Ohki, Jpn. J. Appl. Phys., 49, p-102402, (2010).

[12] H. Hoshina, Y. Morisawa, H. Sato, A.Kamiya, I. Noda, Y. Ozaki andC. Otani, Appl. Phys. Lett., 100, p-101904, (2010).

[13] H. Hoshina, Y.Morisawa, H. Sato, H.Minamide, I. Noda, Y. Ozaki, C. Otani, Phys. Chem. Chem. Phys., 13, p-9173, (2011).

[14] Y.-S. Lee, Principles of Terahertz Science and Technology. New York, NY, USA: Springer, (2009).

[15] K. Kawase, Y. Ogawa, Y. Watanabe and H. Inoue, Opt. Express, 11, p-2549, (2003).

[16] H. Hoshina, Y. Sasaki, A. Hayashi, C. Otani and K. Kawase, Appl. Spectrosc., 63, p-81, (2009).

[17] W. H.Fan, A.Burnett, P. C. Upadhya, J. Cunningham, E. H. Linfield and A. G. Davies, Appl. Spectrosc., 61, p-638, (2007).

[18] K. Fukunaga, Y. Ogawa, S. Hayashi and I. Hosako, IEICE Electron. Express, 4, p-258, (2007).

[19] B. Ferguson and X. C. Zhang, Nature Mater. 1, p-26, (2002).

[20] C. Jansen, S. WietzkeandM. Koch,Springer, 171, p-327, (2013).

[21] S. Wietzke, C. Jansen, M. Reuter, T. Jung, D. Kraft, S. Chatterjee, B.M. Fischer and M. Koch, J. Mol. Structure, 1006, p-41, (2011).

[22] H. Hoshina, Y. Morisawa, H. Sato, A. Kamiya, I. Noda, Y. Ozaki and C. Otani, Appl. Phys. Lett., 96, p-101904, (2010).

[23] H. Hoshina, S. Ishii, Y. Morisawa, H. Sato, I. Noda, Y. OzakiandC. Otani, Appl. Phys. Lett., 100, p011907(1-3), (2012).

[24] H. Hoshina, S. Ishii, S. Yamamoto, Y. Morisawa, H. Sato, T. Uchiyama, Y. Ozaki, and C. Otani, IEEE Transaction, 33, p- 248, (2013).

[25] V. A. Bershtein and V. A. Ryzhov, Adv. Polymer Sci., 114, p-43, (1994).

[26] M. Tasumi and S. Krimm, J.Chem. Phys., 46, p-755, (1967).

[27] M. J. Folkes, A. Keller, J. Stejny, P. L. Goggin, G. V. Fraser andP. J. Hendra, Colloid Polymer Sci., 253, p-354, (1975).

[28] W. Feairhel and J. T.Miller, Appl. Spectrosc., 25, p-175, (1971).

[29] S. C. Shen, L. Santo and L. Genzel, Can. J. Spectrosc., 26, p-126, (1981).

[30] O. F. Nielsen, D.H. Christensen and O. H. Rasmussen, J. Mol. Structure, 242, p-273, (1991).

[31] S. Wietzke, C. Jansen, T. Jung, M. Reuter, B. Baudrit, M. Bastian, S. Chatterjee and M. Koch, Opt. Express, 17, p-19006, (2009).

[32] S. Wietzke, C. Jansen, M. Reuter, T. Jung, J. Hehl, D. Kraft, S. Chatterjee, A. Greiner and M. Koch, Appl. Phys. Lett., 97, 022901(1-3), (2010).

[33] W. F. X. Frank and H. Fiedler, Infrared Phys., 19, p-481, (1979).

[34] M. M. Satkowski, D. H. Melik, J. P. Autran, Physical and Processing Properties of Polyhydroxyalkanoate (PHA) Copolymers. Weinheim: Wiley-VCH, (2001). 
[35] P. A. Holmes, Biologically Produced (R)-3-Hydroxyalkanoate Polymersand Copolymers. London, U.K.: Elsevier, vol. 2, (1988).

[36] H. Sato ,R. Murakami , A.Padermshoke, F. Hirose , K.Senda, I. Noda and Y. Ozaki, Macromolecules, 37, p-7203, (2004).

[37] H. Sato, Y. Ando, J.Dybal, T. Iwata ,I. Noda and Y. Ozaki, Macromolecules, 41, p-4305, (2008).

[38] H. Sato, R. Murakami, J. Zhang, Y. Ozaki, K. Mori, I. Takahashi, H. Terauchi and I. Noda, Macromol. Res., 14, p-408, (2006).

[39] L. Zhang, Z. Zheng, J. Xi, Y. Gao, Q. Ao, Y. Gong, N. Zhao and X. Zhang,Polymer, 48, p-2672, (2007).

[40] S. Yamamoto, Y. Morisawa, H. Sato, H. Hoshina and Y. Ozaki, J. Phys. Chem. B, 117, p-2180, (2013).

[41] M. P. Allen and D. J. Teldesley, Oxford University Press, Oxford, 1987.

[42] H. J. C. Berendsen, J. P. M. Postma and W. F. van Gunsteren,J. Chem. Phys., 81, 3684, (1984).

[43] P. Dauber-Osguthorpe, V.A. Roberts, D.J. Osguthorpe, J. Wolff, M. Genest and A.T. Hagler, Proteins: Struct., Function Genetics, 4, p-31, (1988).

[44] H. J. Sun, Comput. Chem., 15, p-752, (1994).

[45] H. Sun, S. J. Mumby, J. R. Maple and A. T. Hagler, J. Am. Chem. Soc., 116, p-2978, (1994).

[46] H. Sun, P. Ren and J. R. Fried,Comput. Theor. Polym. Sci., 8, p-229, (1998).

[47] A. K. Rappé, C. J. Casewit, K. S. Colwell, W. A. Goddard and W. M. Skiff, J. Am. Chem. Soc., 114, p10024, (1992).

[48] K. F. Khaled and N. S. Abdel-Shafi, Int. Journal of Electrochemi. Soc., 6, p-4077, (2011).

[49] S. L. Mayo, B. D. Olafson and W. A. Goddard, J. Physical Chemistry, 94, p-8897, (1990).

[50] M. Yokouchi, Y.Chatani, H. Tadokoro, K. Teranishi and H. Tani,Polymer, 14, p-267, (1973).

[51] J. Cornibert and R. H. Marchessault, J. Mol. Biol., 71, p-735, (1972).

[52] S. Liu, J. Chang, D. Q. Wei, X. R. Chen, Q. M. Zhang, Z. Z. Gong, G.F. Jiand, Y. X. Guo, J. Atom.Mol. Sci., 3, p-41, (2012). 
